This item was submitted to Loughborough's Research Repository by the author.

Items in Figshare are protected by copyright, with all rights reserved, unless otherwise indicated.

\title{
A review of analytical methods for aircraft structures subjected to high- intensity random acoustic loads
}

PLEASE CITE THE PUBLISHED VERSION

PUBLISHER

Professional Engineering Publishing / @ IMECHE

VERSION

VoR (Version of Record)

LICENCE

CC BY-NC-ND 4.0

\section{REPOSITORY RECORD}

Cunningham, Paul, and R.G. White. 2009. "A Review of Analytical Methods for Aircraft Structures Subjected to High-intensity Random Acoustic Loads”. figshare. https://hdl.handle.net/2134/4872. 
This item was submitted to Loughborough's Institutional Repository (https://dspace.lboro.ac.uk/) by the author and is made available under the following Creative Commons Licence conditions.

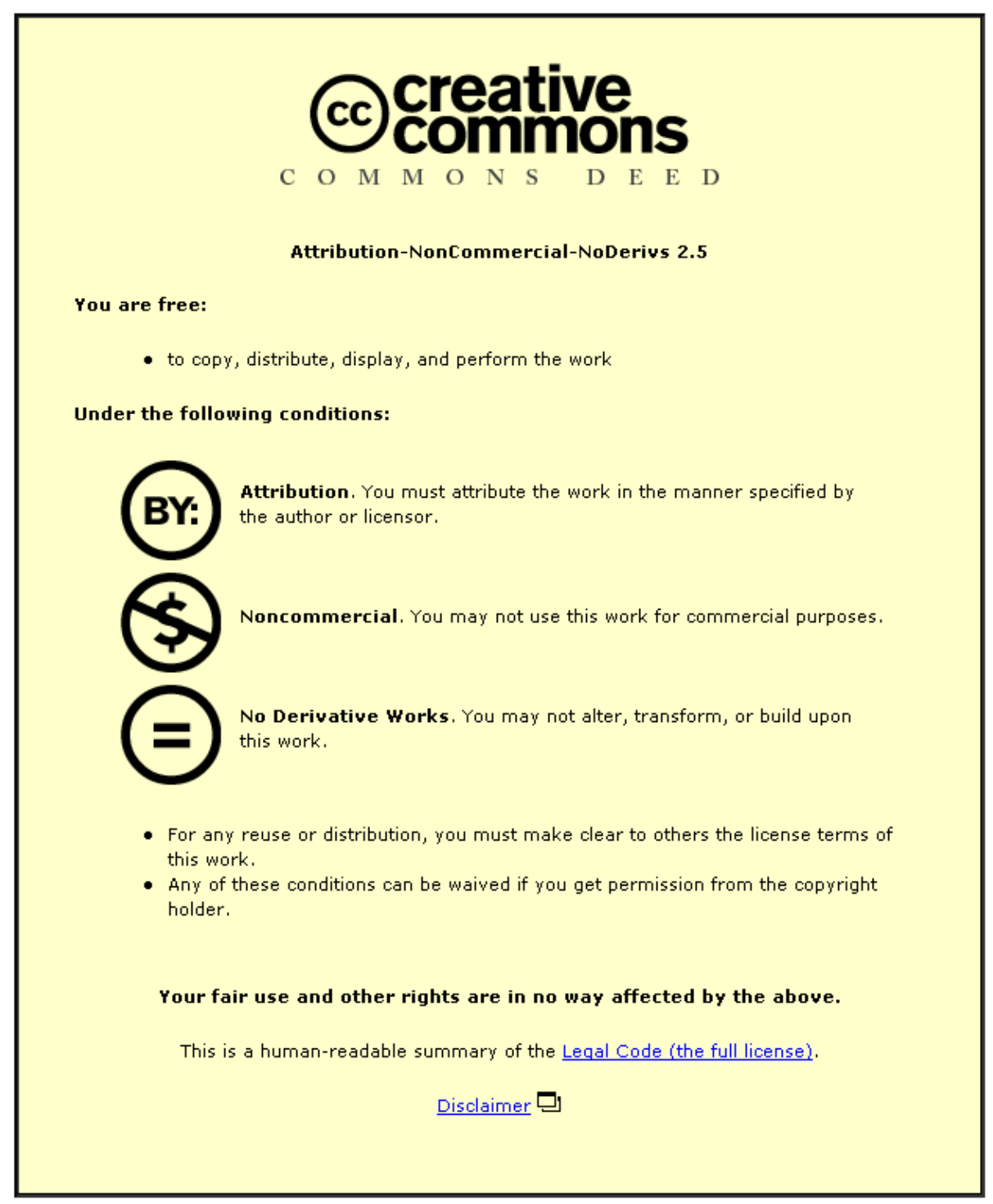

For the full text of this licence, please go to: http://creativecommons.org/licenses/by-nc-nd/2.5/ 


\title{
A review of analytical methods for aircraft structures subjected to high-intensity random acoustic loads
}

\author{
P R Cunningham ${ }^{1 *}$ and $\mathbf{R} \mathbf{G}$ White ${ }^{2}$ \\ ${ }^{1}$ School of Engineering Sciences, University of Southampton, UK \\ ${ }^{2}$ Emeritus Professor of Vibration Studies, University of Southampton, UK
}

\begin{abstract}
A review of the acoustic fatigue design process for aircraft structures is presented in this paper, together with the current design guides, which are used to predict the stresses that an acousticallly loaded aircraft structure may experience in service. These methods are based on linear theory and use the single-degree-of-freedom approximation method. A recent programme of research which uses this method together with the finite element method to predict the root mean square strains experienced by acoustically excited, doubly curved sandwich panels is briefly discussed. Recent developments in prediction methods based on the non-linear dynamic response of thermoacoustic loaded structures are reviewed, and suggestions are made as to possible future directions in the area of acoustic fatigue research.
\end{abstract}

Keywords: acoustic fatigue, aircraft structures, analytical methods

NOTATIO
$c$
$c_{\mathrm{V}}$
$\mathrm{dB}$
$E$
$f$
$F_{\mathrm{n}}$
$G$
$\mathbf{H}$
$\mathrm{i}$
$j$
$J_{\mathrm{n}}$
$k_{\mathrm{a}}$
$m$
$R_{x}$
$R_{y}$
$w, \dot{w}, \ddot{w}$

$x, y, z$
$\omega$
$\phi$
$\rho$
$\mu$

The MS was received on 24 February 2003 and was accepted after revision for publication on 18 September 2003.

* Corresponding author: School of Engineering Sciences-Aerospace Engineering, University of Southampton, Highfield, Southampton, Hampshire S017 1BJ, UK.

$\begin{array}{ll}v & \text { Poisson's ratio } \\ \sigma & \text { direct stress }\left(\mathrm{N} / \mathrm{m}^{2}\right) \\ \psi & \text { spatial distribution of pressure } \\ \zeta & \text { viscous damping ratio } \\ \Re & \text { real part of a complex number } \\ \Im & \text { imaginary part of a complex number }\end{array}$

$\begin{array}{ll}\begin{array}{l}\text { Acronyms } \\ \text { ASTOVL }\end{array} & \begin{array}{l}\text { advanced short take-off and vertical } \\ \text { landing } \\ \text { computational aeroacoustics } \\ \text { carbon-fibre reinforced plastic }\end{array} \\ \text { CFRP } & \text { Engineering Sciences Data Unit } \\ \text { ESDU } & \text { finite element } \\ \text { FE } & \text { finite element analysis } \\ \text { FEA } & \text { overall sound pressure level } \\ \text { OASPL } & \text { progressive wave tube } \\ \text { PWT } & \text { root mean square } \\ \text { r.m.s. } & \text { sound pressure level } \\ \text { SPL } & \end{array}$

\section{INTRODUCTION}

With the introduction of the gas turbine engine and the resulting rapid development of more powerful engines, there followed an increase in the number of reported fatigue failures of aircraft skin structures close to the jet 
exhaust [1]. This led to a range of investigations by several aircraft industries and research establishments in an attempt to both understand and alleviate this problem. The majority of these early investigations involved experimental studies using both simple test panels and actual aircraft structures, and excitation was provided by sirens, random noise generators and aircraft engines. This early work led to the development of the first design nomographs for acoustically excited aircraft structures [2].

A response prediction method for acoustically excited metallic structures was first developed by Miles [3]. In that study, Miles considered an elastic structure subjected to random loading and simplified the analysis by assuming the structure to have only a single degree of freedom. He also used the concept of cumulative damage to analyse the fatigue behaviour of such structures. Miles derived an expression for the equivalent root mean square stress for a single degree of freedom system under random loading. Miner's hypothesis of cumulative damage was then used to estimate the fatigue life of the structure.

Powell [4] took this work one stage further by considering the normal mode approach. The modes of vibration of the structure were assumed to be uncoupled; hence the response of the structure to random pressure loads could be given as a series of $N$ independent equations (one for each mode). Powell developed an expression for the power spectrum of the total stress fluctuation, and in addition he introduced the concept of 'joint acceptance', which is a measure of the effectiveness of the complex pressure field in exciting a particular mode. The cumulative damage law was again used in the estimation of fatigue life.

Following on from Powell's work, Clarkson [5] simplified the theory for panel-type structures by assuming that the major part of the response results from the contribution of one predominant mode. He also assumed that the excitation pressures were exactly in phase over the whole structure and derived an expression for the mean square stress in terms of the viscous damping ratio and resonant frequency:

$$
\overline{\sigma^{2}(t)}=\frac{\pi}{4 \zeta} f_{\mathrm{n}} G_{\mathrm{p}}\left(f_{\mathrm{n}}\right) \sigma_{0}^{2}
$$

where $\sigma_{0}$ is the static stress at the point of interest due to a uniform pressure of unit magnitude, $\zeta$ is the viscous damping ratio and $G_{\mathrm{p}}\left(f_{\mathrm{n}}\right)$ is the excitation pressure spectral density at the resonant frequency $f_{\mathrm{n}}$. This expression was first derived by Miles [3] and is still used today as a design tool for structures subjected to random pressure loading. In order to justify his approach, Clarkson [5] examined the application of the theory to a range of structures, which included flat plates, aircraft control surfaces and integrally stiffened skins, and compared the results with several experi- ments. He concluded that considering the severe simplifications in this theory, the agreement with experimental measurements was satisfactory, and in order to obtain greater accuracy a multi-modal analysis of the structural response would be necessary.

\subsection{Current design guidelines}

The Engineering Sciences Data Unit (ESDU) produces a large amount of design data using the results published in the open literature. The data series covers various aerospace-related disciplines such as aerodynamics, fatigue, dynamics, structures, transonic aerodynamics, performance and vibration and acoustic fatigue. The latter subseries includes work on the prediction of natural frequencies, damping and root mean square (r.m.s.) stresses/strains in various structural elements such as flat and singly curved isotropic and laminated plates, shells and box structures under the action of random acoustic loading [6].

The general procedure adopted in these design guides for estimating the in-service life of composite structures subject to acoustic pressure loading is itemized below.

1. Estimate the orthotropic material properties of the structure from individual ply properties in the lay-up.

2. Estimate the natural frequencies of the structure. This can be done using either ESDU data sheets or the finite element method.

3. Estimate the damping in the structure. Again, ESDU data sheets can be used, but experimental validation is needed. White suggested that a 'bank' of measured data for various types of structure is required [7].

4. Estimate the r.m.s. strain from a knowledge of the fundamental natural frequency, the excitation pressure spectral density at this frequency, the damping and the stress at the point of interest due to a uniform static load, using equation (1).

In the United States, the Acoustic and Vibration Associates produced the 'Sonic fatigue design guide for military aircraft' [8]. Over 300 technical reports, papers, journal publications and textbooks were reviewed to produce the guide, which is a compilation, evaluation, and presentation of existing acoustic fatigue design methods, charts, nomographs and related computer programs. The acoustic source of primary interest is the noise produced by the engine exhaust, although propeller noise, inlet duct noise for high bypass turbofan engines, noise from ducted rotors, ground reflection and aerodynamic noise were all considered. Many structural configurations were investigated in the study, including flat aluminium honeycomb and diffusion bonded titanium honeycomb panels. 


\section{ACOUSTIC FATIGUE OF MODERN AIRCRAFT STRUCTURES}

By the mid to late 1970s there were considerable advances in the design and manufacture of composite structures. Work began to ascertain the effects that random acoustic loading would have on the fatigue life of these new materials, which included boron and glass fibres, Kevlar, and the most promising for aircraft applications - carbon-fibre reinforced plastic (CFRP). One of the earliest studies of the response of CFRP plates to random acoustic loading was made by White [9]. In this paper, comparisons were made between CFRP and aluminium plates and the author showed the non-linear characteristics of the CFRP plates at high excitation levels (above $130 \mathrm{~dB}$ re $2 \times 10^{-5} \mathrm{~Pa}$ ). Nonlinear behaviour was also observed with the aluminium alloy plate; however, this occurred at higher excitation levels compared with the CFRP plate, and the nonlinearity was more pronounced for the CFRP plate studied. This non-linear behaviour was also found during the investigations of Wolfe and Jacobson [10] for tests carried out on a series of multi-bay boronepoxy and graphite-epoxy panels. Although developed for metallic structures, equation (1) was used by White and Mousley [11] to compare experimental measurement of overall plate response with theoretical predictions for CFRP plates. The single-mode predictions using the ESDU data sheets [6], which are based on equation (1), were found to be in very good agreement with the measured values for excitation levels up to 145 dB. Some non-linear effects were observed during this investigation, and the authors went on to study the combination of random acoustic loading and in-plane compression, where the 'snap through' effect was observed [11]. The conclusions drawn indicated that at response levels below those that produce marked nonlinearities, the single-mode method can be used to estimate the r.m.s. response of simple composite plate structures.

In terms of fatigue, the methods developed for metallic structures are not strictly applicable to composite structures. This is because the methods used for metallic structures rely on surface strain estimates, whereas for composite materials it is the combination of internal stresses/strains at a critical point within the layers of the composite, which will cause fatigue damage to initiate and propagate [7]. In addition, the flexural fatigue tests conducted on standard test coupons were found to be unreliable when trying to determine the fatigue life of a specimen, since the specimens were found to delaminate at the edges, as observed by Drew and White [12]. To combat this problem, the half-sine clamp was developed by the authors. This is basically a cantilever clamp with a halfsine wave shape along the clamping edge which, when tested using CFRP test specimens, produced a more representative delamination failure within the test coupon [12].

Soovere investigated the dynamic response of acoustically excited carbon-fibre reinforced and Kevlar honeycomb panels [13]. He extended existing orthotropic honeycomb panel theory, through coupled normal mode analysis, in order to predict the overall strain at both the edges and the centre of the faces of the panels. His work was the first to investigate the effect of the bevelled edge closeout pan, an example of which is shown in Fig. 1, which was shown to increase the effective shear stiffness of the honeycomb core by an order of magnitude. Soovere showed both analytically and experimentally that, with laterally rigid edge supports, the rotation of the bevelled edge introduced a linear dynamic membrane strain into the inner face sheet. He also found that the dominant contribution to honeycomb panel damping was due to acoustic radiation, and for the carbon-fibre reinforced panels, the material damping was very low compared to the Kevlar reinforced panels. For singly curved sandwich panels, it has been suggested that the strains at the centre of the panel are higher on the convex face than on the concave face.

An approximate analytical method for predicting the acoustic fatigue life of plates and shells using multimodal analysis was developed by Blevins [14]. In his paper, Blevins extended Miles' approach [3] to higher modes and complex shapes. He developed methods for estimating the effects of finite acoustic wavelengths on their excitation. Blevins' method is essentially a bridge between deterministic and statistical approaches and his approximations were most applicable to uniform plate and shell structures excited by a stationary (ergodic) sound field. The natural frequencies, mode shapes and the relationship between modal deformation and modal stress are required as inputs in this method. Damping and the magnitude of the applied pressures are also required, although exact knowledge of the distribution of the applied surface pressures is not required, instead an estimate of this distribution was used. For this reason, the concept of joint acceptance was used in Blevins' formulation. Since the evaluation of joint acceptance requires an estimate of the surface acoustic pressure distributions, he suggested several approximations for the shape of these distributions [14]. Blevins used the mass-weighted structural mode shape approximation, from which the joint acceptance was calculated to be unity. An improved approximation for the joint acceptance was developed by using sinusoids to model the structural and acoustic waveforms. A method was then given for calculating the acoustically induced displacements for any mode. In order to validate the method, Blevins compared his results with those produced by the AGARD [15] method, which is a design guide formulated primarily from the work of Miles [3] and Clarkson [5]. A flat plate and a singly 


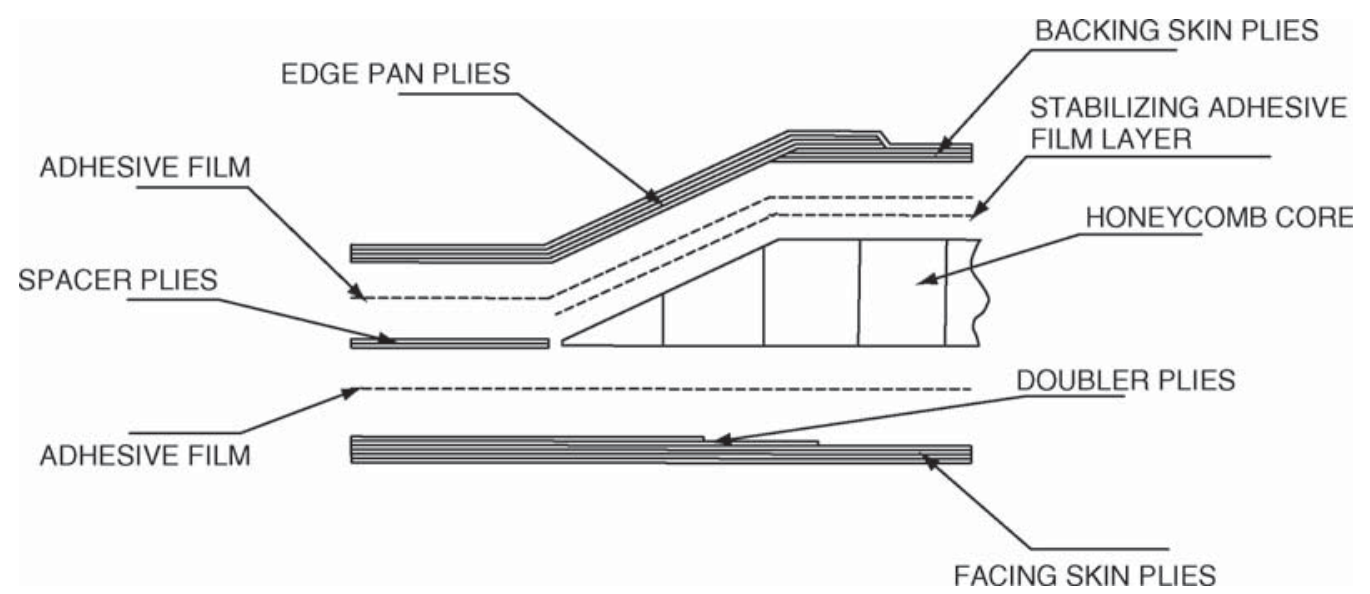

Fig. 1 Bevelled edge detail of the doubly curved honeycomb sandwich panels $[\mathbf{1 6}, \mathbf{1 7}]$

curved plate loaded with broadband sound with an overall sound pressure level of $145.7 \mathrm{~dB}$ were considered. Results from Blevins method agreed very closely with those from the AGARD method and he concluded that the former could be viewed as a generalization of the Miles/Clarkson/AGARD approach. For completeness, Blevins also compared predictions by his method with experimental data obtained from integrally stiffened, flat rectangular titanium panels tested in a progressive wave tube (PWT). The experimental results agreed reasonably well with the theoretical results within the bounds of uncertainty.

\section{RECENT DEVELOPMENTS}

\subsection{Response prediction of acoustically excited, doubly curved honeycomb sandwich panels}

A recent programme of research at the University of Southampton investigated the dynamic response prediction of acoustically excited, doubly curved honeycomb sandwich panels $[\mathbf{1 6}, \mathbf{1 7}]$. The panels were designed with a constant plan area of $0.912 \mathrm{~m} \times 0.525 \mathrm{~m}$ to fit the test aperture of the PWT facility. Three radii of curvature designs were employed; $R_{x}=3.5 \mathrm{~m} \times R_{y}=1.0 \mathrm{~m}, R_{x}=$ $1.2 \mathrm{~m} \times R_{y}=1.0 \mathrm{~m}$ and $R_{x}=3.5 \mathrm{~m} \times R_{y}=0.5 \mathrm{~m}$, with $x$ being the long side dimension and $y$ being the short side dimension. Three 'symmetric sandwich' panels and one 'asymmetric sandwich' panel (with $R_{x}=3.5 \mathrm{~m} \times R_{y}=$ $0.5 \mathrm{~m}$ ) were manufactured, giving a total of four test panels. The panel design can be broken into four main sections; the facing skin which forms the inner face of the panel, the core, the edge pan plies which enclose the edge of the core and form the attachment flange and bevelled edge (shown in Fig. 1), and the backing skin which forms the outer face of the panel. This design is typical of that used in the construction of aircraft-type panels such as intake barrel panels and flap fairing panels, and a typical test panel drawing can be seen in Fig. 2.

Each panel was secured in the test aperture of the PWT using four circular steel springs. This was done to ensure that the lower resonant response frequencies were within the $60-600 \mathrm{~Hz}$ bandwidth (this is a bandwidth limitation of the PWT siren), and the highest possible strains were achieved in the centre of the panels. In addition, the panels and associated boundary conditions in the PWT could be easily modelled using finite element analysis (FEA) with this configuration. Strain measurements were taken at various locations on both faces of the panels, and comparisons were made between r.m.s. strain at sound pressure levels from 143 to $164 \mathrm{~dB}$ (re $2 \times 10^{-5} \mathrm{~Pa}$ ) over a frequency band from 60 to $600 \mathrm{~Hz}$. The panels were manufactured using plain weave carbon cloth and an over expanded paper honeycomb core [18]. The panel design incorporates a bevelled edge which is typical of in-service aircraft honeycomb panels, as shown in Fig. 1.

The specifications for the four test panels used in this investigation are given in Table 1 and a complete description of the panels, including the manufacture process, can be found in reference [18].

\subsubsection{The single-degree-of-freedom (SDOF) approximation method}

In order to predict the dynamic response of the acoustically excited, doubly curved honeycomb sandwich panels, two approaches were adopted. Firstly, the single-degree-of-freedom (SDOF) approximation method was used, which is the classical approach used to predict the response of plate-type structures to random acoustic loading and is based on the method originally developed by Miles [3], and subsequently refined by Powell [4] and Clarkson [5]. At low frequencies, the modes of vibration of a structure are generally well separated in frequency, and assuming the modal damping is small, approximations can be made to 


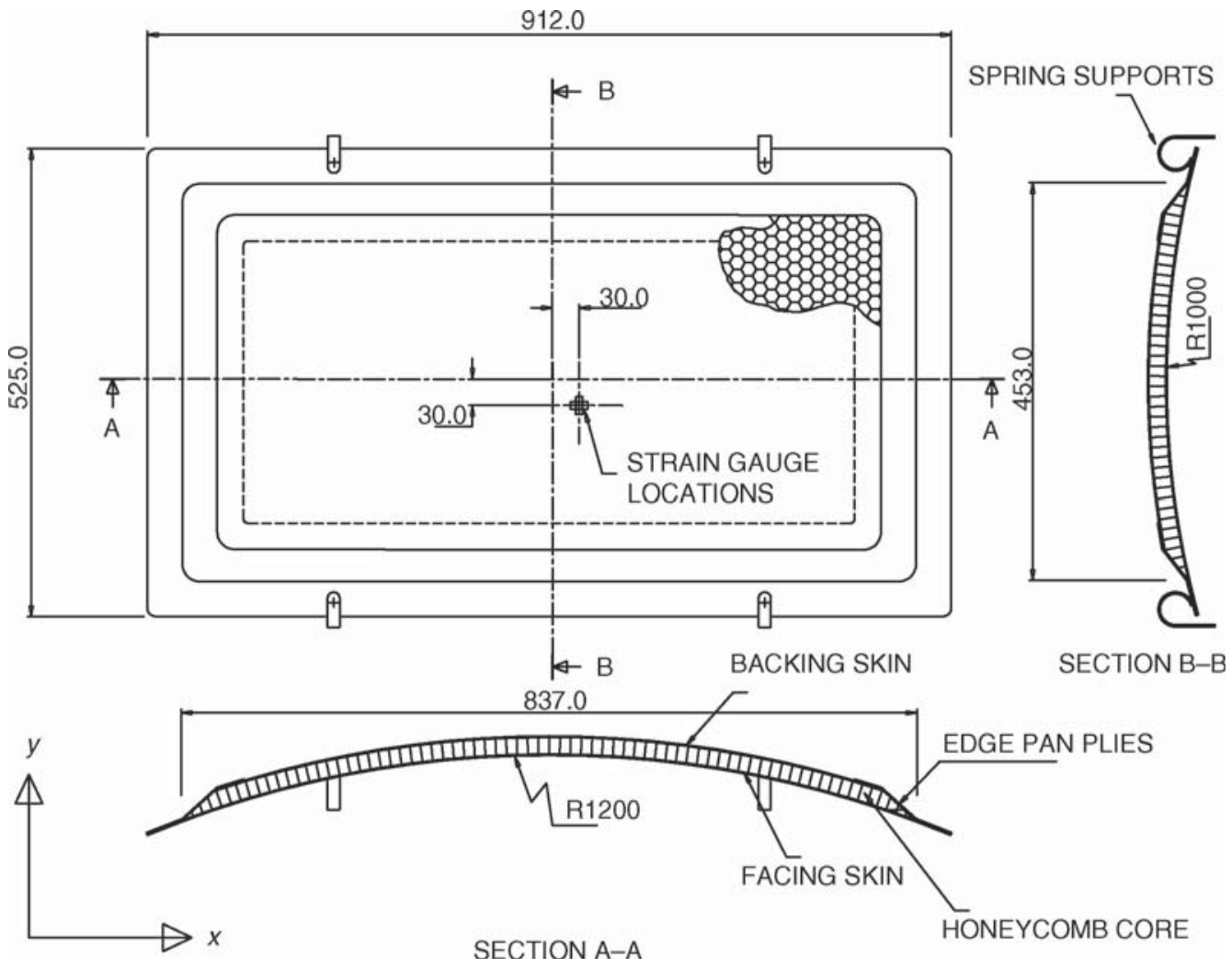

Fig. 2 A typical test panel drawing showing the spring support locations for the doubly curved sandwich panel investigation $[\mathbf{1 6}, \mathbf{1 7}]$

Table 1 Dimensions, ply lay-up and material specifications for the doubly curved test panels $[\mathbf{1 6}, \mathbf{1 7}]$

\begin{tabular}{|c|c|c|c|c|}
\hline Dimensions (m) & Panel 1 & Panel 2 & Panel 3 & Panel 4 \\
\hline$x$ & 0.912 & 0.912 & 0.912 & 0.912 \\
\hline$y$ & 0.525 & 0.525 & 0.525 & 0.525 \\
\hline$R_{x}$ & 3.5 & 1.2 & 3.5 & 3.5 \\
\hline \multirow[t]{2}{*}{$R_{y}$} & 1.0 & 1.0 & 0.5 & 0.5 \\
\hline & \multicolumn{2}{|c|}{ Panels 1, 2 and 3} & \multicolumn{2}{|c|}{ Panel 4} \\
\hline Layer & Number of plies & $\theta$ & Number of plies & $\theta$ \\
\hline Facing skin & 4 & {$[0 / 45]_{\mathrm{s}}$} & 2 & \\
\hline Doublers & 2 & {$[0 / 30]^{\mathrm{s}}$} & 2 & {$[0 / 30]$} \\
\hline Spacers & 2 & {$[0]_{2}$} & 2 & \\
\hline Edge pan & 3 & {$[0 / 45 / 0]$} & 3 & {$[0 / 45 / 0]$} \\
\hline Backing skin & 4 & {$[0 / 45]_{\mathrm{s}}$} & 6 & {$[0 / 45 / 0]$} \\
\hline Properties & \multicolumn{2}{|l|}{ CFRP } & \multicolumn{2}{|l|}{ Honeycomb } \\
\hline Thickness (m) & \multicolumn{2}{|l|}{$0.25 \times 10^{-3}$} & \multicolumn{2}{|l|}{$19.0 \times 10^{-3}$} \\
\hline$E_{x}(\mathrm{~Pa})$ & \multicolumn{2}{|l|}{$57.93 \times 10^{9}$} & \multicolumn{2}{|l|}{0} \\
\hline$E_{y}(\mathrm{~Pa})$ & \multicolumn{2}{|l|}{$57.93 \times 10^{9}$} & \multicolumn{2}{|l|}{0} \\
\hline$E_{z}(\mathrm{~Pa})$ & \multirow{2}{*}{\multicolumn{2}{|c|}{$\overline{37} \times 10^{9}$}} & \multicolumn{2}{|l|}{$120 \times 10^{6}$} \\
\hline$G_{x y}(\mathrm{~Pa})$ & & $3.7 \times 10^{9}$ & \multicolumn{2}{|l|}{0} \\
\hline$G_{x z}(\mathrm{~Pa})$ & \multirow{2}{*}{\multicolumn{2}{|c|}{ - }} & \multirow{2}{*}{\multicolumn{2}{|c|}{$\begin{array}{l}35 \times 10^{6} \\
20 \times 10^{6}\end{array}$}} \\
\hline$G_{y z}(\mathrm{~Pa})$ & & & & \\
\hline$\rho\left(\mathrm{kg} / \mathrm{m}^{3}\right)$ & \multicolumn{2}{|l|}{1518.0} & \multicolumn{2}{|l|}{48.0} \\
\hline$v_{x y}$ & \multicolumn{2}{|l|}{0.04} & \multicolumn{2}{|l|}{ - } \\
\hline
\end{tabular}


model each mode of vibration as a SDOF system:

$$
\ddot{w}(t)+2 \zeta_{\mathrm{n}} \omega_{\mathrm{n}} \dot{w}(t)+\omega_{\mathrm{n}}^{2} w(t)=F_{\mathrm{n}}(t)
$$

where $F_{\mathrm{n}}(t)$ is the generalized force. Assuming $F_{\mathrm{n}}(t)=\mathrm{e}^{\mathrm{i} \omega t}$ and $w(t)=\mathbf{H}(\omega) \mathrm{e}^{\mathrm{i} \omega t}$, then the substitutions $\dot{w}(t)=\mathrm{i} \omega \mathbf{H}(\omega) \mathrm{e}^{\mathrm{i} \omega t}$ and $\ddot{w}(t)=-\omega^{2} \mathbf{H}(\omega) \mathrm{e}^{\mathrm{i} \omega t}$ can be made in equation (2) to give

$$
\left[-\omega^{2}+\left(2 \zeta_{\mathrm{n}} \omega_{\mathrm{n}}\right) \mathrm{i} \omega+\omega_{\mathrm{n}}^{2}\right] \mathbf{H}(\omega)=1
$$

where $t$ is the time, i is a complex number and $\mathbf{H}(\omega)$ is the frequency response function.

A graphical representation of the SDOF estimation method [19] can be seen in Fig. 3. The approximate calculation of the response of the SDOF system to broadband random loading with a constant spectral density can be made by approximating the area under the curve of $|\mathbf{H}(\omega)|^{2}$ with a rectangle of the same area [19]. As can be seen, at resonance the peak value of $|\mathbf{H}(\omega)|^{2}$ is $1 /\left(c_{v}^{2} \omega_{\mathrm{n}}^{2}\right)=1 /\left(4 \zeta_{\mathrm{n}}^{2} k_{\mathrm{s}}^{2}\right)$ and the bandwidth is approximated by $\pi \zeta_{\mathrm{n}} \omega_{\mathrm{n}}$ since the stiffness $k_{\mathrm{s}}=\omega_{\mathrm{n}}^{2} m$ and the viscous damping $c_{v}=2 \zeta_{\mathrm{n}} \omega_{\mathrm{n}} m$. For the approximation to be valid, the excitation bandwidth must be reasonably broadband and include $\omega_{\mathrm{n}}$, and the damping should be light with the natural frequencies of vibration being well spaced [19]. The mean square response $\sigma_{\mathrm{w}}^{2}$ is simply the product of the input spectral density, the mean square bandwidth and the peak of the frequency response function, $|\mathbf{H}(\omega)|^{2}$. This leads to

$$
\sigma_{\mathrm{w}}^{2}=\frac{\pi G_{\mathrm{n}}(\omega)}{4 \zeta_{\mathrm{n}} \omega_{\mathrm{n}}^{3}}
$$

where $G_{\mathrm{n}}(\omega)$ is the input random excitation spectrum, which is defined by both the spatial and temporal aspects of the excitation pressure. By assuming the pressure field has separable spatial and temporal characteristics, which is true for certain pressure fields such as travelling waves, and taking the spatial average over this pressure field, the spectrum of the pressure, $p(t)$, can be written in terms of the pressure measured at a single point for homogeneous pressure fields. This leads to an expression for the excitation pressure spectrum, $G_{\mathrm{n}}(\omega)$ as follows:

$$
G_{\mathrm{n}}(\omega)=\frac{G_{\mathrm{p}}(\omega)}{\left\langle\psi^{2}(\boldsymbol{x})\right\rangle_{\boldsymbol{x}}}\left[\frac{\int \psi(\boldsymbol{x}) \phi_{\mathrm{n}}(\boldsymbol{x}) \mathrm{d} \boldsymbol{x}}{\int m \phi_{\mathrm{n}}^{2}(\boldsymbol{x}) \mathrm{d} \boldsymbol{x}}\right]^{2}
$$

where $G_{\mathrm{p}}(\omega)$ is the measured pressure spectrum, $\phi_{\mathrm{n}}(\boldsymbol{x})$ is the response mode shape obtained from the eigenvectors and $m$ is the mass per unit area. It therefore remains to specify $\psi(x)$ in order to fully define the broadband random excitation spectrum $G_{\mathrm{n}}(\omega)$.

Three different cases were considered for $\psi(\boldsymbol{x})$. Case 1 was the assumption of a uniform pressure distribution, $\psi(\boldsymbol{x})=1$, which is the assumption used by Miles [3] and Clarkson [5]. Case 2 was the assumption that the spatial characteristics of the pressure loading exactly matched the response mode shape, $\psi(\boldsymbol{x})=\phi_{\mathrm{n}}(\boldsymbol{x})$, which is equivalent to stating that the joint acceptance function equals unity, and case 3 was the assumption that the spatial distribution of the loading, $\psi(\boldsymbol{x})$, is assumed to be neither uniform or equal to the mode shape, $\phi_{\mathrm{n}}(\boldsymbol{x})$. For the latter case, an expression for the joint acceptance was formulated by assuming that the pressure loading could be considered as travelling acoustic waves along the axis of the PWT aperture. Assuming that the waves are unidirectional travelling along the axis of the duct, the spatial distribution of the

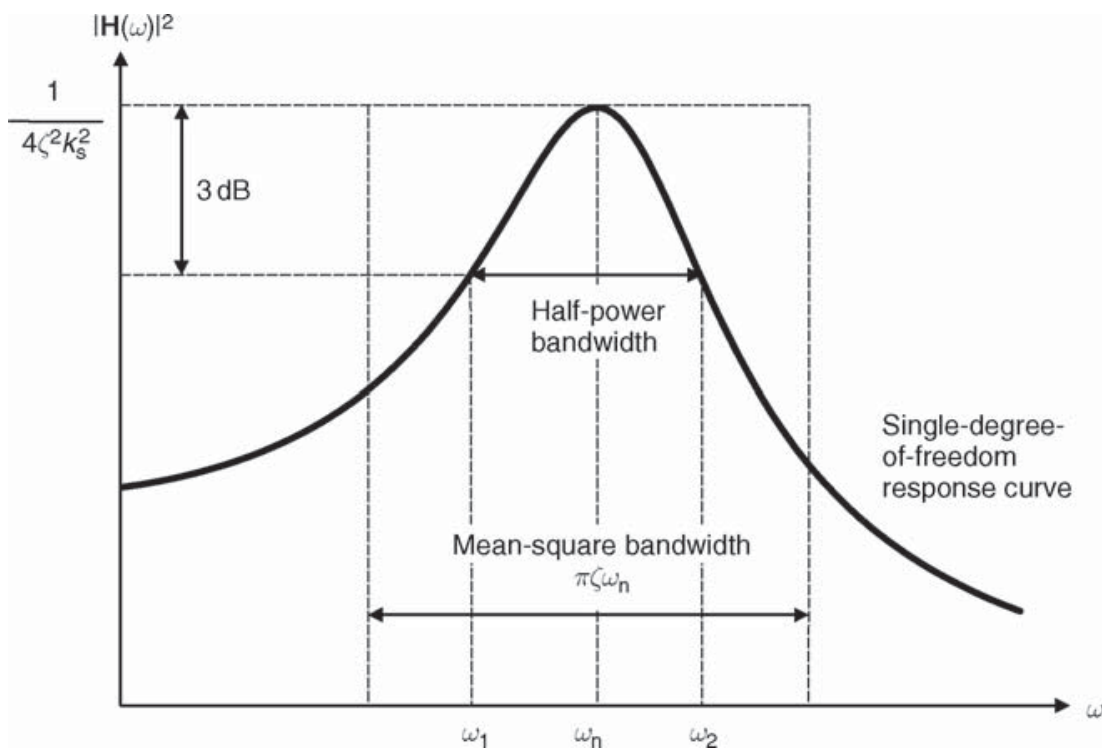

Fig. 3 Estimation of the mean-square response of a single-degree-of-freedom system with broadband random excitation $[\mathbf{1 6}, \mathbf{1 7}]$ 
pressure loading can be written in the form

$$
H(x, f)=\mathrm{e}^{-\mathrm{i} k_{\mathrm{a}} x}
$$

where $k_{\mathrm{a}}=\omega_{\mathrm{n}} / c$ is the acoustic wavenumber, $\omega_{\mathrm{n}}$ is the natural frequency of vibration of the relevant mode of the test panel $\left(\omega_{\mathrm{n}}=2 \pi f_{\mathrm{n}}\right)$ and $c$ is the speed of sound, which is approximately $343 \mathrm{~m} / \mathrm{s}$ at sea level with standard atmospheric conditions, and $x$ is the position along the long side dimension of the panel. A rapid estimate of the joint acceptance could therefore be obtained by using the results for the mode shape from an FEA modal analysis of the panels, a typical result of which is shown in Fig. 4 for panel 1. By summing the

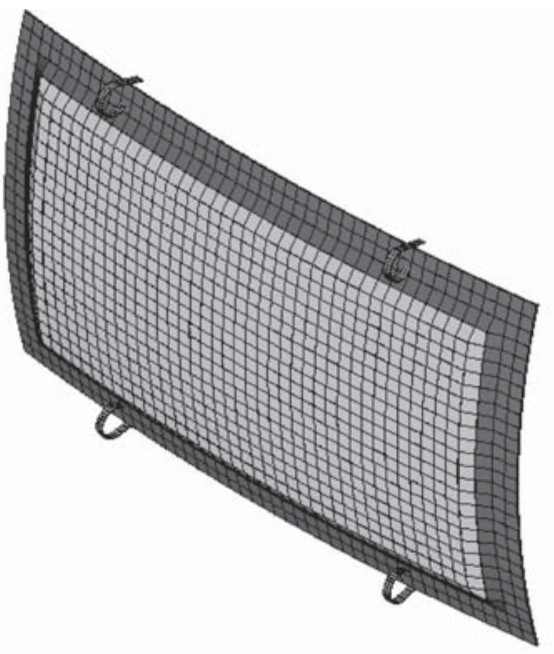

(a) FE model of panel 1

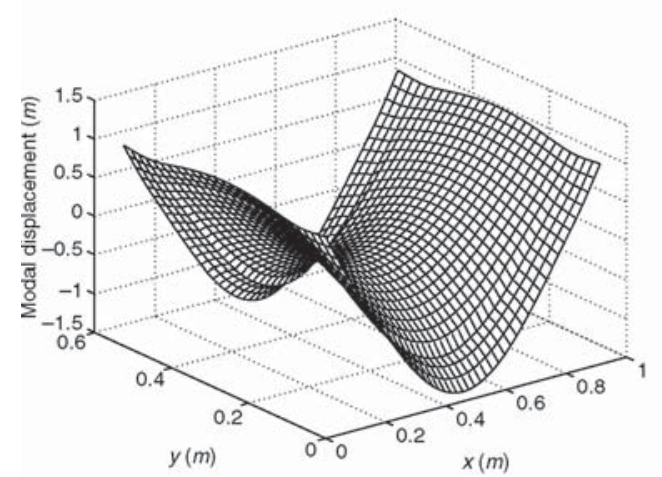

(b) Panel 1 modal displacement

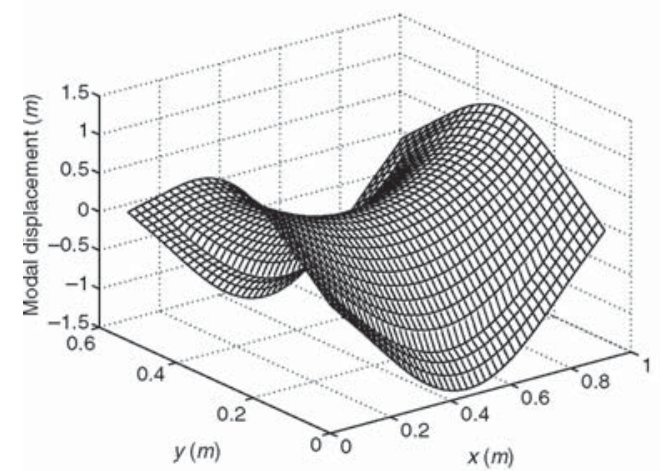

(d) Panel 3 modal displacement

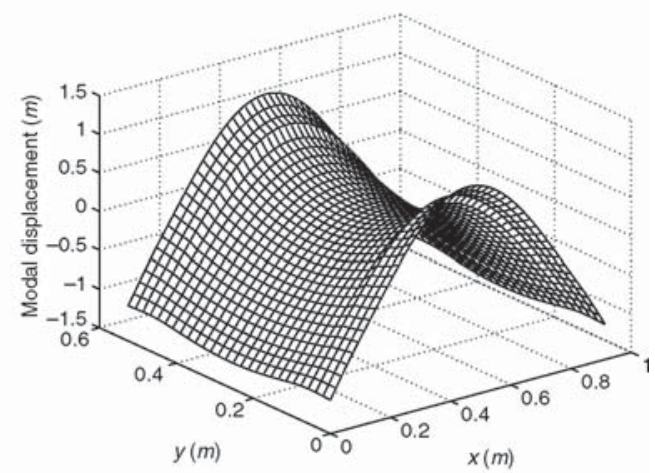

(c) Panel 2 modal displacement

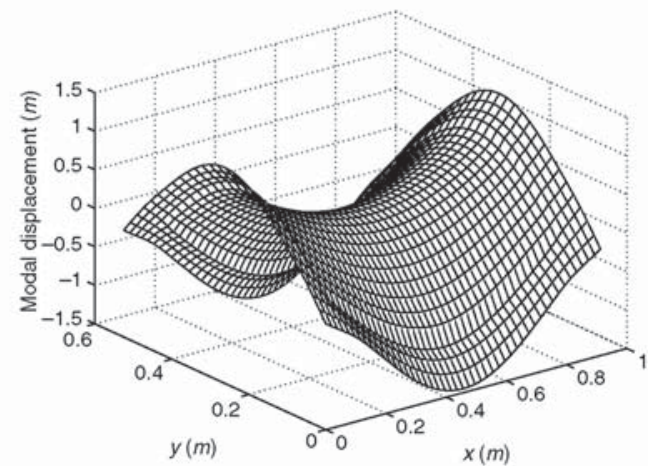

(e) Panel 4 modal displacement

Fig. 4 FE model of panel 1 and calculated modal displacement associated with the fundamental frequency of vibration excited by the PWT for the four doubly curved test panels $[\mathbf{1 6}, \mathbf{1 7}]$ 
modal displacemements for the mode shape in question and multiplying by the area of the panel divided by the number of nodes, an estimate of the integral of the mode shape is obtained. Thus the joint acceptance function used was [17]

$$
J_{\mathrm{n}}^{2}=\frac{\left|\sum_{j=1}^{N}\left\{\phi_{\mathrm{n}}\right\}_{j} \mathrm{e}^{-\mathrm{i} k_{\mathrm{a}} x_{j}}\right|^{2}}{\sum_{j=1}^{N}\left|\mathrm{e}^{-\mathrm{i} k_{\mathrm{a}} x_{j}}\right|^{2} \sum_{j=1}^{N}\left\{\phi_{\mathrm{n}}\right\}_{j}^{2}}
$$

where $\left\{\phi_{\mathrm{n}}\right\}_{j}$ is the modal displacement at each node $j$ for the mode shape in question, $x_{j}$ is the nodal coordinate in the $x$ direction (along the long side of the panel) for each node $j$ and $N$ is the number of nodes in the FEA model.

The results from the three cases of the SDOF approximation method are shown in Fig. 5. For case 1 , the results are shown to be significantly lower than the measured strains. The reason for the very low theoretical prediction can be traced to the fact that the panel is located in the PWT aperture by using circular steel springs. This arrangement leads to a mode of vibration that is almost a rigid-body translation, and which therefore has almost the same spatial distribution as a uniform pressure load. This mode is orthogonal to all the higher modes of the panel, and thus the integral of a higher mode shape multiplied by a constant will be almost zero. Thus a uniform pressure load produces almost no excitation of the mode selected for the theoretical prediction (the first bending mode), and a very small strain is predicted. The problem with the prediction is not that the wrong mode has been selected but rather that the actual pressure loading is very different from a uniform distribution.
For case 2, where the spatial distribution of the pressure loading is assumed to match the mode shape exactly, the results show that this assumption leads to an overestimate of the response compared with the scaled measured strains. This is understandable since the assumption that the spatial distribution of the pressure load matches exactly the mode shape of the panel is unlikely in practice. This assumption was investigated further by Blevins [14], and it can be seen that case 1 (a rigid-body displacement of the panel) and case 2 (unit joint acceptance, where the joint acceptance varies between 0 and 1) provide the lower and upper bounds on the response [17].

The r.m.s. strain results for case 3, obtained by employing equation (7), show excellent agreement between measured and predicted values. This is a very favourable result when it is considered that the current design guidelines are reported to give results within a factor of 2 compared with measured data [1].

\subsubsection{The finite element model}

The approach taken to predict the dynamics response using the FEA method was to model a series of travelling waves over the whole surface of the panel within the frequency range of interest. The analysis was divided into $10 \mathrm{~Hz}$ frequency bands from 50 to $500 \mathrm{~Hz}$ in which the acoustic wavenumber, $k_{\mathrm{a}}$, was held constant. A harmonic analysis was carried out in each frequency band with five substep calculations made, centred on the frequency step in the loop. Within each of these frequency bands, the loading was applied in lines that ran the length of the short side and were spaced at intervals equal to the element edge length from one end

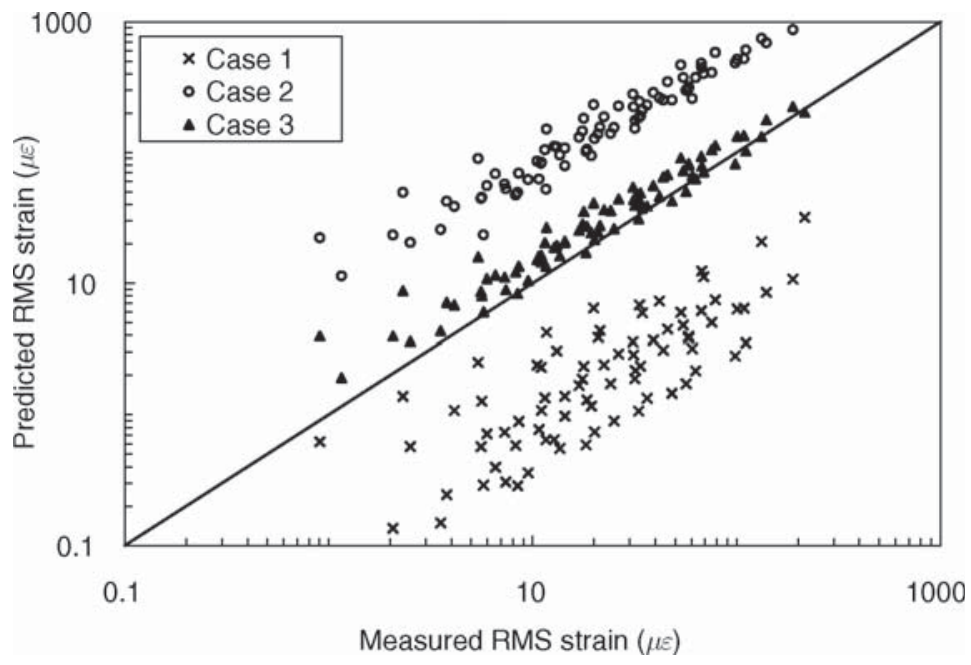

Fig. 5 Comparison of measured and predicted r.m.s. strains using the SDOF approximation method [16, 17]. Case $1, \psi(\boldsymbol{x})=1$, uniform pressure distribution. Case $2, \psi(\boldsymbol{x})=\phi_{\mathrm{n}}(\boldsymbol{x})$, spatial distribution of pressure exactly matches structural mode shape. Case $3, \psi(\boldsymbol{x}) \neq \phi_{\mathrm{n}}(\boldsymbol{x})$, spatial distribution of pressure neither uniform nor equal to the mode shape 


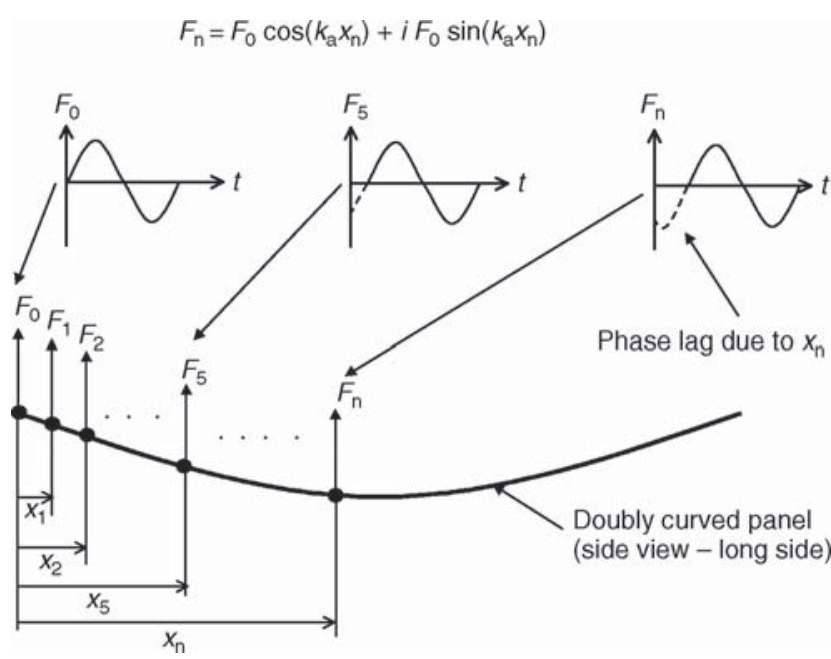

Fig. 6 Illustration of the simulated travelling wave loading applied to the FEA model $[\mathbf{1 6}, \mathbf{1 7}]$

of the panel to the other along the long side. Unit forces were applied at the corner nodes on these lines, and the real and imaginary parts of the load were defined according to the $x_{\mathrm{n}}$ location of the nodal line and the frequency step being analysed, which along with the convection velocity, $c=342.8 \mathrm{~m} / \mathrm{s}$, defined the acoustic wavenumber, $k_{\mathrm{a}}$. Hence,

$$
\Re\left\{F_{\mathrm{n}}\right\}=F_{0} \cos \left(k_{\mathrm{a}} x_{\mathrm{n}}\right)
$$

and

$$
\Im\left\{F_{\mathrm{n}}\right\}=F_{0} \sin \left(k_{\mathrm{a}} x_{\mathrm{n}}\right)
$$

where $F_{0}=1 \mathrm{~N}$ and $k_{\mathrm{a}}=2 \pi f / c$. In this way, a travelling wave was simulated, as illustrated in Fig. 6. It can be seen that in progressing along the panel (in the $x$ direction) each line of nodal harmonic forces has a phase that will lag behind the previous line of nodal forces according to the distance from the edge of the panel, i.e. at $x=0$. Therefore, for each harmonic solution where the frequency is kept constant, a travelling wave is simulated by virtue of this phase difference between adjacent lines of nodal force. The results from the model were output in terms of the strain transfer function (in $\mu \varepsilon / \mathrm{N}$ ) versus the substep frequency values for each strain gauge location.

From this, the strain PSD was obtained by first calculating the strain transfer function in terms of pressure, taking the absolute value and squaring, before finally multiplying by the measured pressure spectrum, $G_{\mathrm{p}}(f)$, obtained from the experiments

$$
G_{\varepsilon}=\left|\frac{\mathbf{H}_{\varepsilon}(f)}{\left(F_{0} N / A\right)}\right|^{2} G_{\mathrm{p}}(f)
$$

where $\mathbf{H}_{\varepsilon}(f)$ is the strain transfer function obtained from the FEA model (in terms of force), $N$ is the total number

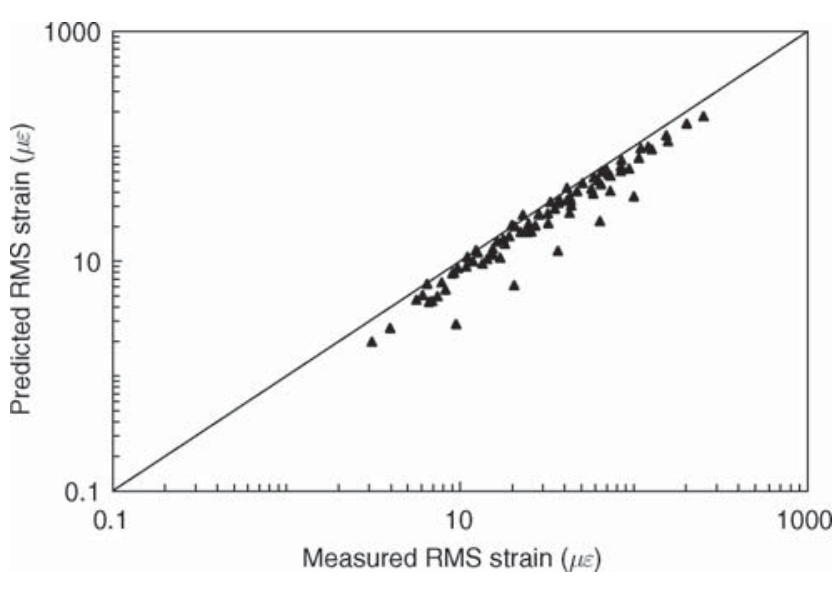

Fig. 7 Comparison of measured and predicted r.m.s. strains using the FEA harmonic analysis method [16, 17]

of nodes on which the unit force, $F_{0}$, was applied, and $A$ is the panel surface area.

The results of the FEA are shown in Fig. 7. The r.m.s. strain levels were calculated and compared with the measured r.m.s. responses over the same frequency bandwidth $(50-500 \mathrm{~Hz})$. As can be seen, the FEA method resulted in a consistent underpredicted r.m.s. strain response.

An example of the comparison between FEA and measurement over the entire frequency bandwidth is shown in Fig. 8. In the FEA, the first four non-rigid body modes were included in the harmonic response and damping values for each of these modes were included. It is therefore unclear why higher modes are not as apparent in the FEA results compared with the measured response. However, the agreement between the experimental results and predicted response was very good for the fundamental mode in all cases. One possible explanation for the higher mode disagreement could be that in the PWT there was some asymmetric excitation across the width of the panel (i.e. from the top to the bottom of the aperture), which could have been enough to excite the higher modes that have nodal lines running along the length of the panel. Since the excitation in the travelling wave method adopted in the FE calculations was constant in phase across the width of the panel ( $y$ direction), any modes with nodal lines along the length of the panel would not have been excited. In any case, it is clear from the FEA results that the travelling wave method works well for analysing the response of structures to random acoustic excitation.

\subsection{Prediction and measurement of the non-linear response due to higher intensity/thermoacoustic loading}

There has been increasing interest over the last few years on the prediction and measurement of the non-linear 


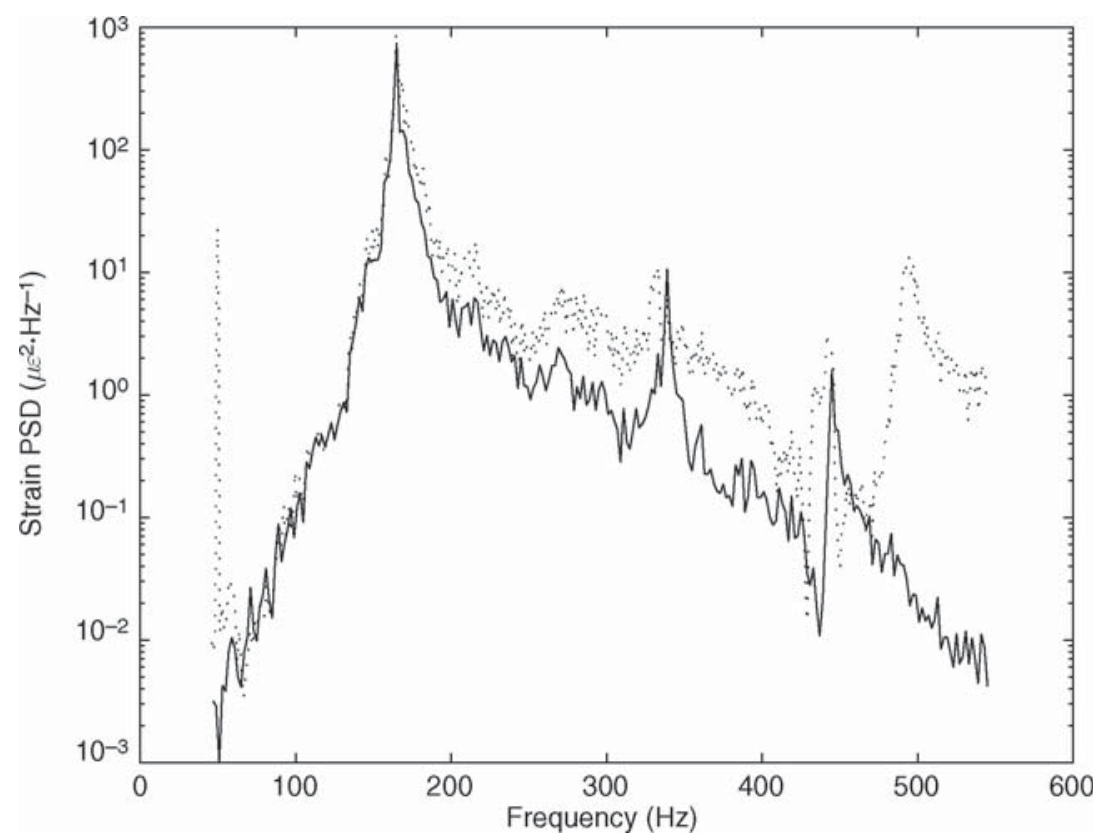

Fig. 8 Comparison of predicted FE (-) and measured (- -) strain PSD for the inner centre gauge ( $x$-wise), panel $2[\mathbf{1 6}, \mathbf{1 7}]$

response of structures due to high intensity loading. This has been driven, in part, by the increasing sound pressure levels being predicted and experienced by the latest generation of ASTOVL strike fighters, coupled with greater use of lighter, stiffer composite structures. In addition, with advanced stealth technology, stores are being located internally, which is presenting a new problem in the form of cavity noise and the high intensity loads which are being experienced within the bays that house the stores of these aircraft. In conjunction with higher sound pressure levels, recent developments in acoustic fatigue research have also concentrated on combined thermal-acoustic environments, which are being driven by studies of possible hypersonic vehicle designs [20, 21].

Green and Killey [22] highlighted the fact that the current adopted method of using linear vibration theory for determinig fatigue life, which assumes that the pressure field is fully correlated over the surface area of the structure, becomes increasingly unsatisfactory when geometric non-linearities occur due to high sound pressure levels. They also noted that the excitation will generally be out-of-phase over the structure due to complex aerodynamic effects [22]. A time domain Monte Carlo (TDMC) technique was used to model the multi-modal vibrations of aircraft structures, which was implemented by interfacing with a conventional FEA code. Analyses were carried out for a simple flat plate with and without combined loads (i.e. high intensity acoustic pressures plus static loads), and the results indicated that the overall predicted stress levels were better than those between predictions based on linear or equivalently linear theory and test [22]. The downside of the approach was that the TDMC runs took a significant amount of computer time to carry out [22], but another advantage was that the 'snap-through' phenomenon can be modelled using the TDMC/FEA approach, which is not possible with existing methods. Other work in this area includes that of Rizzi and Muravyov [23], who used the equivalent linearization (EL) approach to analyse the random vibration of geometrically non-linear multiple-degree-of-freedom structures. The results from this analysis, which is a statistical linearization method, were compared with results from an FE numerical analysis. The authors [23] noted that the EL method has potential advantages over conventional linear analyses when computing the fatigue life based on r.m.s. stress levels; however, this method requires careful investigation when conducting a fatigue life calculation based on the EL-derived PSD response [23]. Finally, for a non-linear response prediction, McEwan et al. [24] extended an FE-based modal approach to the case of multi-modal, non-linear beam vibrations. The method was used to model isotropic beams with simply supported and fully clamped boundary conditions, with free vibration and steady state harmonic excitation considered. The work showed good agreement compared with a standard direct integration FE approach, with considerable savings in computational expense.

For the case of combined thermoacoustic loading, recent studies include that of Holehouse [21] who reported on an experimental and analytical programme concerned with the structural response and fatigue characteristics of skin panels for a generic hypersonic flight vehicle. Carbon-carbon, silicon-carbide refactory 
composites, titanium metal-matrix composites and advanced titanium alloys were investigated, and FE thermal and sonic fatigue analyses were conducted to predict the response. High-temperature random fatigue shaker tests were used to determine the fatigue strength at high temperatures, and high-temperature acoustic fatigue tests (in a PWT) were conducted to compare with the predicted response [21]. The results indicated a good level of agreement between FE and the test results, although the actual method of analysis used was not clear. Other investigations of thermoacoustic loaded structures include those of Lee and Wentz [25], who used a single-mode Fokker-Planck formulation to predict the high-temperature response of thermally buckled plates, Udrescu [26], who used a higher-order FE method to model the non-linear vibration of thermally buckled plates, and Dhainaut et al. [27], who used an FEA time-domain modal formulation to predict the non-linear random response of composite panels subjected to accoustic pressures at elevated temperatures.

\section{POSSIBLE FUTURE DEVELOPMENTS}

It is evident that there is renewed interest in the dynamic response of aircraft structures subjected to high-intensity acoustic loads. The majority of the present effort is aimed at predicting the non-linear response of aircraft structures under combined loading at high-intensity pressure levels. However, the authors would like to suggest other avenues that warrant further investigation in the light of this resurgent interest. These are:

1. There is a need for more experimental data for acoustically excited aircraft structures. This is crucial if the advances in the prediction methods are to be validated, and should include investigations at high levels up to $175 \mathrm{~dB}$ (re $2 \times 10^{-5} \mathrm{~Pa}$ ) for combined static-acoustic and thermoacoustic loadings. A wide range of structural configurations and materials should be included to give a broad range of data in both the linear and non-linear response regimes for comparison with the prediction methods. In addition, these tests should be performed using progressive wave tubes, reverberation chambers and wind tunnels to give a complete representation of the environment in which these structures must perform.

2. The advances currently being made in the prediction methods are very encouraging. However, there is still a need for greater accuracy, both in terms of the predicted structural response and the representation of the acoustic loads. More work is needed in interfacing computational aeroacoustic (CAA) codes with finite element analysis or other structural prediction methods. In addition, a complete representation of the response of composites is needed in terms of failure analysis, since it is the stresses within critical regions of the lay-up that will cause failure in most cases and not only stresses at the surface of the material [7]. This will no doubt require a huge computational effort; therefore the application of idealized models or substructuring could help to reduce this expense.

3. In addition to the response prediction, there is a need for more accurate predictions of fatigue life of structures subject to high-intensity acoustic excitation. This includes a more detailed assessment of the fatigue of structures under combined static-acoustic and thermoacoustic loadings, and should encompass the wide variety of structures and materials mentioned previously.

\section{CONCLUSIONS}

In this paper, a brief review has been conducted on the dynamic response prediction of aircraft structures to high-intensity acoustic loads. This has included a review of the acoustic fatigue design process, current design guides such as the ESDU/AGARD approach and recent progress in the response prediction of doubly curved sandwich structures to acoustic excitation. The methods presented in the latter investigation are similar to those adopted by ESDU/AGARD and others such as Blevins [14], and have led to good agreement between measured and predicted r.m.s. strains for this type of aircraft structure.

Both linear and non-linear response prediction methods have been covered, and the relative merits and shortfalls of these methods have been discussed. It is clear that there is a resurgent interest in the acoustic fatigue design process, which is being driven by a combination of increasing sound pressure levels (SPL), greater use of composite materials and interest in hypersonic vehicle designs. This has led to a greater emphasis on non-linear thermoacoustic response prediction methods, a selection of which have been reviewed here.

Finally, an attempt has been made to highlight possible areas that require attention with regard to the acoustic fatigue design process in the future. The main point here is an increase in the amount of test data needed to support the advances in response prediction methods. In addition, there is a need to know, and to be able to predict, the spatial characteristics of random pressure fields on all regions of aircraft structures and from a variety of sources such as engine fans, jet efflux and turbulent boundary layers. This could be achieved using techniques such as computational fluid dynamics, or more specifically computational aeroacoustics. If used in conjunction with the finite element method, this 
would provide the designer with a very powerful response prediction tool.

\section{ACKNOWLEDGEMENT}

The authors would like to acknowledge the Engineering and Physical Sciences Research Council (EPSRC), who funded the work concerned with the response prediction of acoustically excited, doubly curved sandwich structures under Grant GR/K80600.

\section{REFERENCES}

1 Clarkson, B. L. Review of sonic fatigue technology. NASA Technical Report CP-4587, 1994.

2 McGowan, P. R. Structural design for acoustic fatigue. Technical Report TDR-63-820, ASD, October 1963.

3 Miles, J. W. On structural fatigue under random loading. J. Aeronaut. Sci., 1954, 21, 753-762.

4 Powell, A. On the fatigue failure of structures due to vibrations excited by random pressure fields. J. Acoust. Soc. Am., 1958, 30(12), 1130-1135.

5 Clarkson, B. L. Stresses in skin panels subjected to random acoustic loading. Aeronaut. J.R. Aeronaut. Soc., 1968, 72, $1000-1010$

6 Engineering Sciences Data Unit Design Guide Series: Vibration and Acoustic Fatigue, Vols 1-7, August 2001, Supplement level 48 (ESDU International, London).

7 White, R. G. Developments in the acoustic fatigue design process for composite aircraft structures. Composite Structs, 1990, 16, 171-192.

8 Rudder, F. F. and Plumblee, H. E. Sonic fatigue design guide for military aircraft. Technical Report AFFDL-TR74-112, Acoustics and Vibration Associates, Atlanta, Georgia, Febrary 1975.

9 White, R. G. A comparison of some statistical properties of the responses of aluminium alloy and CFRP plates to acoustic excitation. Composites, 1978, 9, 251-258.

10 Wolfe, N. D. and Jacobson, M. J. Design and sonic fatigue characteristics of composite material components. In Symposium on Acoustic Fatigue, Toulouse, France, 1972, AGARD Conference Proceedings 113.

11 White, R. G. and Mousley, R. F. Dynamic response of CFRP plates under the action of random acoustic loading. In Proceedings of the Fourth International Conference on Composite Structures, Paisley College of Technology, 1987, pp. 1519-1535.

12 Drew, R. C. and White, R. G. An experimental investigation into damage propogation and its effects upon dynamic properties in CFRP composite material. In Proceedings of the Fourth International Conference on Composite Structures, Paisley College of Technology, 1987, pp. 245-256.

13 Soovere, J. Random vibration analysis of stiffened honeycomb panels with beveled edges. Am. Inst. Aeronaut, Astronaut. J. Aircr., 1986, 23(6), 537-544.

14 Blevins, R. D. An approximate method for sonic fatigue analysis of plates and shells. J. Sound Vibr., 1989, 129(1), $51-71$.

15 Thompson, A. G. R. and Lambert, R. F. The estimation of RMS stresses in stiffened skin panels subjected to random acoustic loading. Technical Report Section 5, AGARDAG-162, Advisory Group for Aerospace Research and Development, NATO, November 1972.

16 Cunningham, P. R. and White, R. G. Dynamic response prediction of doubly curved honeycomb sandwich panels to random acoustic excitation. Part 1: experimental study. J. Sound Vibr., July 2003, 264(2), 579-603.

17 Cunningham, P. R., Langley, R. S. and White, R. G. Dynamic response prediction of doubly curved honeycomb sandwich panels to random acoustic excitation. Part 2: theoretical study. J. Sound Vibr., July 2003, 264(2), 605637.

18 Cunningham, P. R., White, R. G. and Aglietti, G. S. The effects of various design parameters on the free vibration of doubly curved composite sandwich panels. J. Sound Vibr., 2000, 230(3), 617-648.

19 Norton, M. P. Fundamentals of Noise and Vibration Analysis for Engineers, 1996 (Cambridge University Press, Cambridge).

20 Blevins, R. D., Holehouse, I. and Wentz, K. R. Thermoacoustic loads and fatigue of hypersonic vehicle skin panels. J. Aircr., 1993, 30, 971-978.

21 Holehouse, I. Sonic fatigue characteristics of high temperature materials and structures for hypersonic vehicle applications. In Proceedings of the 6th International Conference on Recent Advances in Structural Dynamics, University of Southampton, July 1997, Vol. 2, pp. 935-949.

22 Green, P. D. and Killey, A. Time domain dynamic finite element modelling in acoustic fatigue design. In Proceedings of the 6th International Conference on Recent Advances in Structural Dynamics, University of Southampton, July 1997, Vol. 2, pp. 1007-1026.

23 Rizzi, S. A. and Muravyov, A. A. Comparison of nonlinear random response using equivalent linearization and numerical simulation. In Proceedings of the 7th International Conference on Recent Advances in Structural Dynamics, University of Southampton, July 2000, Vol. 2, pp. 833-846.

24 McEwan, M. I., Wright, J. R., Cooper, J. E. and Leung, A. Y. T. A combined modal/finite element analysis technique for the dynamic response of a non-linear beam to harmonic excitation. J. Sound Vibr., 2001, 243(4), 601-624.

25 Lee, J. and Wentz, K. R. Strain power spectra of a thermally buckled plate in random vibration. In Proceedings of the 6th International Conference on Recent Advances in Structural Dynamics, University of Southampton, July 1997, Vol. 2, pp. 903-917.

26 Udrescu, R. Nonlinear vibrations of thermally buckled panels. In Proceedings of the 7th International Conference on Recent Advances in Structural Dynamics, University of Southampton, July 2000, Vol. 2, pp. 757-768.

27 Dhainaut, J. M., Duan, B., Mei, C., Spottswood, S. M. and Wolfe, H. F. Non-linear response of composite panels to random excitations at elevated temperatures. In Proceedings of the 7th International Conference on Recent Advances in Structural Dynamics, University of Southampton, July 2000, Vol. 2, pp. 769-784. 